\title{
Framework of Monitoring Patient Safety Culture by the Bootstrap Method
}

\author{
Chih-Hsuan Huang ${ }^{\mathrm{a}, \mathrm{b}}$, Yii-Ching Lee ${ }^{\mathrm{c}, \mathrm{d}}$, and Hsin-Hung $\mathrm{Wu}^{\mathrm{e}, \mathrm{f}, *}$ \\ ${ }^{a}$ School of Business Administration, Hubei University of Economics, Wuhan, 430062, China \\ ${ }^{b}$ Institute for Development of Cross-Strait Small and Medium Enterprise, Hubei University of Economics, Wuhan, 430205, China \\ ${ }^{c}$ Department of Health Business Administration, Hung Kuang University, Taichung, 43302, China \\ ${ }^{d}$ School of Health Policy and Management, Chung Shan Medical University, Taichung, 40201, China \\ ${ }^{e}$ Department of Business Administration, National Changhua University of Education, Changhua, 50007, China \\ ${ }^{f}$ Department of M-Commerce and Multimedia Applications, Asia University, Taichung, 41354, China
}

\begin{abstract}
This study proposes a framework to monitor the patient safety culture by means of a safety attitudes questionnaire by using the bootstrap method to determine the performance of each dimension of safety attitudes questionnaire in each year based on a small sample size. The focus is not the bootstrap method itself but the philosophy of using the bootstrap method to construct the control chart-like limits to observe the performance of each dimension based on the small sample size. Hospital management can better understand how each dimension performs on a yearly basis from the data available. When more new data are available, the boundaries generated by the bootstrap method can be adjusted. Besides, the trends and changes for each dimension can be traced in a control chart that enables hospital management to observe how the patient safety culture changes from time to time. This study demonstrates the philosophy of using the bootstrap method when the sample size is seven for each dimension. The trends and changes for each dimension can be observed for hospital management by construction its control chart.
\end{abstract}

Keywords: bootstrap method; patient safety culture; safety attitudes questionnaire; key performance index; trend analysis

(Submitted on October 13, 2018; Revised on April 15, 2019; Accepted on May 10, 2019)

(C) 2019 Totem Publisher, Inc. All rights reserved.

\section{Introduction}

Patient safety culture is increasingly given importance in hospitals due its positive contributions to performance, such as patient satisfaction, patient loyalty, healthcare quality, and lower medical errors [1-4]. The safety attitudes questionnaire (SAQ) developed by Sexton et al. [5] has been adopted widely to evaluate patient safety culture in healthcare organizations [6-8].

Monitoring staff's attitudes toward patient safety culture can regularly help hospital management understand current conditions and subsequently improve unsatisfied services or nonideal patient safety culture dimensions [8-9]. Lee et al. [10] suggested that regularly assessing safety culture is essential to monitor the changes as well as trends in a healthcare organization. Despite the fact that patient safety culture has been assessed yearly by hospitals in Taiwan, whether the value for each dimension is relatively high or low can only be determined by analyzing the current year's data. In contrast, hospital management may be more interested in realizing improvements and monitoring trends in patient safety culture by using a longitudinal comparison study. However, it is not easy for a hospital to collect sufficient data in a short period of time to monitor the changes and trends in patient safety culture for each dimension in the SAQ, as the survey is usually conducted only once a year.

In each regional hospital or medical center in Taiwan, more than one hundred key performance indices are monitored each day. To effectively identify abnormal conditions on a daily basis, hospital management needs to establish an incident

\footnotetext{
* Corresponding author.

E-mail address: hhwu@cc.ncue.edu.tw
} 
reporting system to first identify and then remove the adverse events and injuries in the organizations [11]. With the progress in computing and statistical technologies, the bootstrap method allows researchers to create sufficient data based on a small amount of raw data to infer a population when the population is unknown [12-13]. Thus, hospital management can monitor the changes on a timely basis even when only a small amount of raw data is available. For instance, the philosophy of control limits can be established to observe the perceptions of patient safety culture from hospital medical staffs.

The boundary lines of each dimension of the SAQ can be calculated to allow hospital management to assess the performance when a limited number of observations is available, through random sampling with replacement to construct confidence intervals [14]. That is, when new numbers of dimensions of the SAQ are available each year through interval surveys, hospital management can easily detect if any dimension has a relatively low perception, indicating that a particular dimension may be worsening. Thus, an action can be taken to improve patient safety culture on a timely basis when a small amount of data is available for analyses.

The purpose of this study is to propose a framework of applying the bootstrap method to use resampling from the limited available sample data from the SAQ annually and infer a population when the population is unknown. More importantly, the estimates of confidence intervals derived by the bootstrap method can be constructed and used as upper and lower limits for hospitals to monitor the performance of patient safety culture on a timely basis. This framework would enable hospitals to detect changes and trends as quickly as possible when a new survey shows the dimensions of patient safety culture are perceived to have worsened.

\section{Literature Review}

\subsection{Patient Safety Culture}

Patient safety culture in healthcare organizations has become one of the most essential ways to improve medical services and minimize patient harm continuously from the processes of care delivery [9, 15-16]. Positive culture result in several benefits for healthcare organizations [8, 17-18]. For example, Johari et al. [17] suggested that hospital employees with a better attitude toward patient safety could reduce medical errors and enhance patient satisfaction. Lee et al. [8] concluded that hospitals that emphasize safety culture more would lower adverse events including patient falls, medical errors, and work absence. Therefore, it is essential for hospitals to regularly evaluate frontline staff's attitudes toward patient safety to allow hospital management to realize patient safety in the healthcare process and the cues to improve the atmosphere of safety culture.

The widely used SAQ has 30 questions, grouped into six dimensions: teamwork climate (the perceived quality of collaboration between personnel), safety climate (the perceptions of a strong and proactive organizational commitment to safety), job satisfaction (the positivity regarding the work experience), stress recognition (the measurement of how performance is influenced by stressors), perception of management (the approval of managerial actions), and working conditions (the perceived quality of the work environment and logistical support such as staffing and equipment) [5, 19]. The details of each question for measuring these six dimensions can be found in Lee et al. [9]. A five-point Likert's scale is applied to each question that ranges from "strongly agree" to "strongly disagree." In addition, two reversed questions are designed to further be more accurate in wording. The negatively worded questions are reverse scored so that their valence matches the positively worded questions. The scores for each of the six dimensions are calculated by summing the scores of the items measuring that particular dimension. That is, there are 6, 7, 5, 4, 4, and 4 questions summed to measure teamwork climate, safety climate, job satisfaction, stress recognition, perceptions of management, and working conditions, respectively.

\subsection{Bootstrap Method}

The bootstrap method was introduced by Efron and is defined as the resampling procedure for assessing the statistical distributions based on independent observations [20]. Several statistical measures (e.g., p-values for statistical tests and confidence intervals) could be performed by a simulation without knowing the underlying probability distributions [14, 21].

The bootstrap method is one of the common techniques employed for illustrating the effect of uncertain sample statistics on probabilistic or reliability models. In particular, the bootstrap method is adopted for the unknown distribution of a statistical parameter and/or the insufficient sample of size for inferring statistical implications [22-23]. The applications by recognizing the sampling properties of various statistics can be found in geotechnical and structural engineering [24-26], economic growth and financial development [27-28], and consumption behavior [29-30].

The philosophy of the bootstrap method is to use only what we know from the data without introducing extraneous 
assumptions about the population distribution [31]. When $F$ is the population distribution and $T(F)$ is the function that defines the parameter with a sample size $n$, the bootstrap distribution $F_{n}^{*}$ plays the role of $F_{n}$ in the resampling process.

The original sample is a sample of $n$ independent identically distributed observations from the distribution $F$, and the sample estimate of the parameter is $T\left(F_{n}\right)$. That is, $F_{n}$ is the empirical distribution, randomly sampling with replacement from the original data. The exact bootstrap estimate of the parameter is computed by averaging appropriately over all possible bootstrap samples theoretically. If we randomly generate larger bootstrap samples, the distribution of bootstrap estimates will approximate the bootstrap distribution for the estimate [31].

\section{Research Method}

A framework of using the bootstrap method is proposed to examine the changes and trends for each dimension of patient safety culture by using resampling from the limited sample data available from the SAQ annually. The estimates of confidence intervals derived by the bootstrap method can be developed as references to detect if the current performance (current raw data) is relatively poor compared with the lower limit. In this study, the algorithms of the bootstrap method and confidence intervals are not the focal point; the philosophy of using the bootstrap method to monitor the changes and trends in patient safety culture is. Therefore, we use MATLAB built-in functions to generate the needed data for illustration.

The built-in functions for the bootstrap method in this study are depicted below.

$$
\begin{aligned}
& \text { bootstat }=\text { bootstrp (nboot, bootfun, } \mathrm{d} 1, \cdots) \\
& {[\text { bootstat, bootsam }]=\text { bootstrp }(\cdots)}
\end{aligned}
$$

Where bootstat $=$ bootstrp(nboot, bootfun, $\mathrm{d} 1, \cdots)$ draws nboot bootstrap data samples, computes statistics on each sample using bootfun, and returns the results in the matrix bootstat. In addition, nboot must be a positive integer, and bootfun is a function handle specified with @. Each row of bootstat contains the results of applying bootfun to one bootstrap sample. If bootfun returns a matrix or array, then this output is converted to a row vector for storage in bootstat. The third input arguments $(\mathrm{d} 1, \cdots)$ are data (scalars, column vectors, or matrices) used to create inputs to bootfun. That is, bootstrp creates each bootstrap sample by sampling with replacement from the rows of the nonscalar data arguments (these must have the same number of rows). Moreover, bootfun accepts scalar data unchanged. [bootstat, bootsam] $=$ bootstrp $(\cdots)$ returns an n-by-nboot matrix of bootstrap indices, bootsam. Each column in bootsam contains indices of the values drawn from the original data sets to constitute the corresponding bootstrap sample (https://www.mathworks.com/help/stats/bootstrp.html?s tid=srchtitle).

\section{Case Illustration for the Proposed Framework}

A regional teaching hospital in Taichung City, Taiwan was chosen as an example for illustration. To efficiently set a baseline of assessing the patient safety culture in the hospital, the average values of six dimensions from 2010 to 2016 were calculated based on the annual SAQs. Physicians and nurses in the hospital were asked to answer the questionnaire by conducting an intraorganizational (interval) online survey. The score for each dimension was obtained by aggregating the scores of the questions in that particular dimension. The average values of the six dimensions of the SAQs from 2010 to 2016 are summarized in Table 1.

Table 1. Scores of six dimensions from 2010 to 2016

\begin{tabular}{|c|c|c|c|c|c|c|}
\hline Dimension & $\begin{array}{c}\text { Teamwork } \\
\text { climate }\end{array}$ & $\begin{array}{c}\text { Safety } \\
\text { climate }\end{array}$ & $\begin{array}{c}\text { Perceptions of } \\
\text { management }\end{array}$ & $\begin{array}{c}\text { Job } \\
\text { satisfaction }\end{array}$ & $\begin{array}{c}\text { Stress } \\
\text { recognition }\end{array}$ & $\begin{array}{c}\text { Working } \\
\text { conditions }\end{array}$ \\
\hline 2010 & 21.473 & 24.408 & 17.071 & 14.674 & 13.038 & 13.446 \\
\hline 2011 & 22.414 & 24.061 & 18.249 & 14.470 & 14.048 & 14.274 \\
\hline 2012 & 20.689 & 24.133 & 17.566 & 14.652 & 13.549 & 13.855 \\
\hline 2013 & 20.970 & 24.479 & 17.768 & 14.906 & 13.696 & 13.860 \\
\hline 2014 & 22.332 & 24.995 & 17.879 & 14.126 & 13.697 & 13.753 \\
\hline 2015 & 22.686 & 25.673 & 18.005 & 14.473 & 13.809 & 14.027 \\
\hline
\end{tabular}

Each dimension was sampled 10,000 times, and the values of upper control limit (UCL), center line (CL), and lower control limit (LCL) for each dimension are computed by 99.865 percentile, average value, and 0.135 percentile, respectively. The MATLAB code for teamwork climate, for example, is provided below. 
clear;

format short

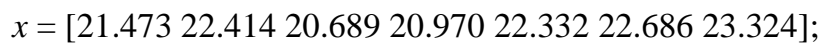

[bootstat, bootsam] = bootstrp $(10000, @(x)[x], x)$;

$y=\operatorname{bootstat}(1: 10000,:)$;

Maxvalue $=\max (y,[], 2)$;

$\mathrm{UCL}=$ prctile $($ Maxvalue, $99.865,1)$

Avgvalue $=\operatorname{mean}(y, 2)$;

$\mathrm{CL}=$ mean (Avgvalue)

Minvalue $=\min (y,[], 2)$;

$\mathrm{LCL}=$ prctile $($ Minvalue, $0.135,1)$

The notations of UCL, CL, and LCL are respectively the upper control limit, center line, and lower control limit values obtained after resampling 10,000 times. The UCL, CL, and LCL values for each dimension are depicted in Table 2.

Table 2. The upper control limit, center line, lower control limit values generated by the bootstrap method

\begin{tabular}{|c|c|c|c|c|c|c|}
\hline Timension & $\begin{array}{c}\text { Teamwork } \\
\text { climate }\end{array}$ & Safety climate & $\begin{array}{c}\text { Perceptions of } \\
\text { management }\end{array}$ & $\begin{array}{c}\text { Job } \\
\text { satisfaction }\end{array}$ & $\begin{array}{c}\text { Stress } \\
\text { recognition }\end{array}$ & $\begin{array}{c}\text { Working } \\
\text { conditions }\end{array}$ \\
\hline UCL & 23.3240 & 26.1110 & 18.5390 & 14.9060 & 14.3940 & 14.3730 \\
\hline CL & 21.9847 & 24.8389 & 17.8699 & 14.5090 & 13.7473 & 13.9417 \\
\hline LCL & 20.6890 & 24.0610 & 17.0710 & 14.1260 & 13.0380 & 13.4460 \\
\hline
\end{tabular}

Hospital management can assess the performance for each dimension in a straightforward manner by comparing the actual score and control limits. For instance, if the score of a particular dimension in 2016 is close to UCL value provided in Table 2, it indicates that the physicians and nurses perceive that dimension well. If the score of a particular dimension is within the UCL and LCL values, it indicates that the perceptions of that dimension are normal. However, if the score of a particular dimension is near the LCL value, it is a warning that the perceptions of that dimension are worsening. In this study, the perceptions of teamwork climate, safety climate, perceptions of management, stress recognition, and working conditions by physicians and nurses are close to the UCL values, showing that patient safety culture has been improved for these dimensions. On the other hand, the score of job satisfaction is within the UCL and LCL values, indicating that the job satisfaction perceived by physicians and nurses in 2016 is acceptable and normal. By further comparing the score with the average value, the score is found to be within the CL and LCL values, showing that the performance of job satisfaction needs to be monitored because this value is closer to LCL rather than the center line.

In addition, control charts philosophy can be applied for hospital management to observe the trends and changes from year to year. Then, hospital management can observe how the patient safety culture changes from time to time in terms of dimensions and what efforts should be invested to improve the patient safety culture in the hospital. Figures 1 to 6 provide the trends of teamwork climate, safety climate, perceptions of management, job satisfaction, stress recognition, and working conditions by control charts philosophy, respectively. Though five dimensions except for job satisfaction have the patterns going upward, teamwork climate, perceptions of management, stress recognition, and working conditions (Figures 1, 3, 5, and 6) fluctuate from the lowest point to the highest point. In contrast, the trend of safety climate seems to steadily increase. On the other hand, the performance of job satisfaction (Figure 4) is in a normal condition. However, the trend seems to be decreasing. Therefore, improving job satisfaction for physicians and nurses may be in a high priority to enhance the patient safety culture in this case hospital.

For traditional statistical approaches to evaluate the safety culture, hospital management needs to collect data for more than seven years to perform data analyses. In contrast, the framework based on the bootstrap method enables hospitals to track the changes and trends in patient safety culture on a timely basis even when the sample of size is very limited. In hospitals, patient safety is critically important. Assessing the changes in safety culture helps management understand how the hospital performs and what actions should be taken to improve patient safety, and to provide a better place for both patients and medical staff. Moreover, when the data in 2017 are available, the UCL, CL, and LCL values for each dimension can be computed by using the data from 2010 to 2017. It is worth noting that the UCL, CL, and LCL values can be updated quickly to better reflect the safety culture. Finally, the philosophy of this framework can be applied to similar key performance indices in any hospital worldwide to quickly provide the snapshots for hospital management. 


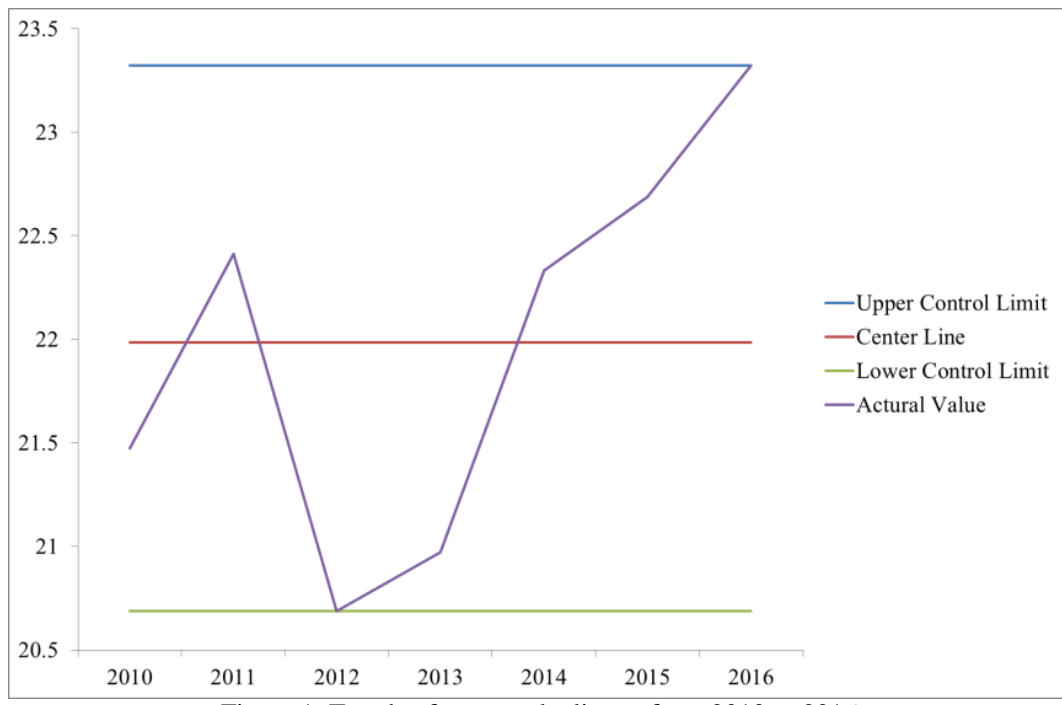

Figure 1. Trends of teamwork climate from 2010 to 2016

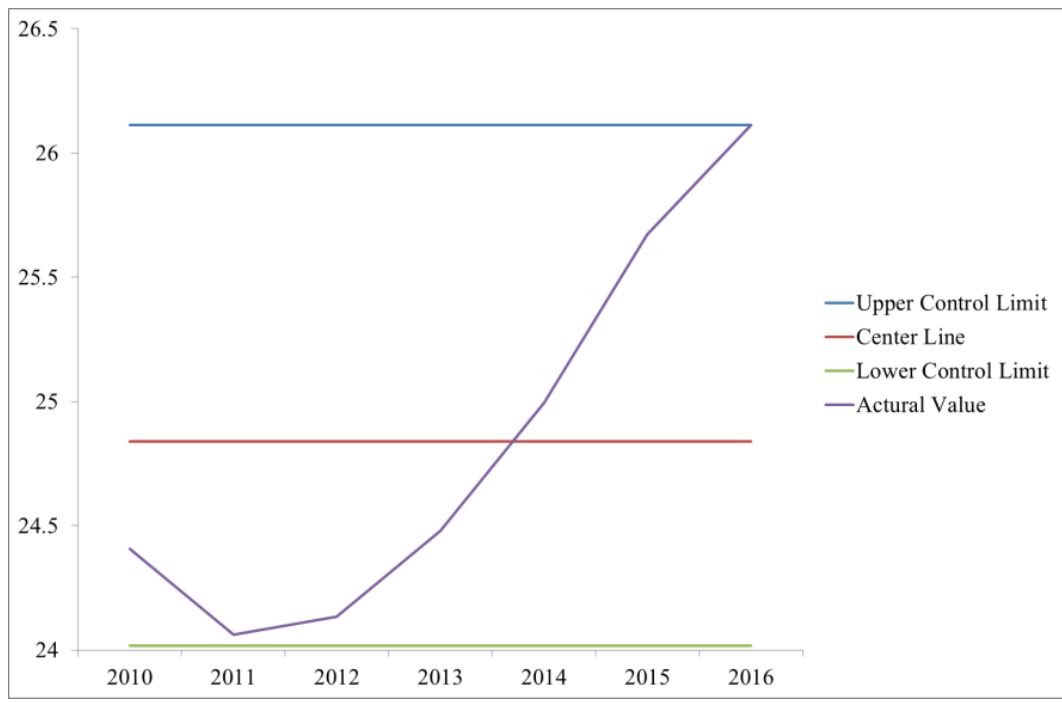

Figure 2. Trends of safety climate from 2010 to 2016

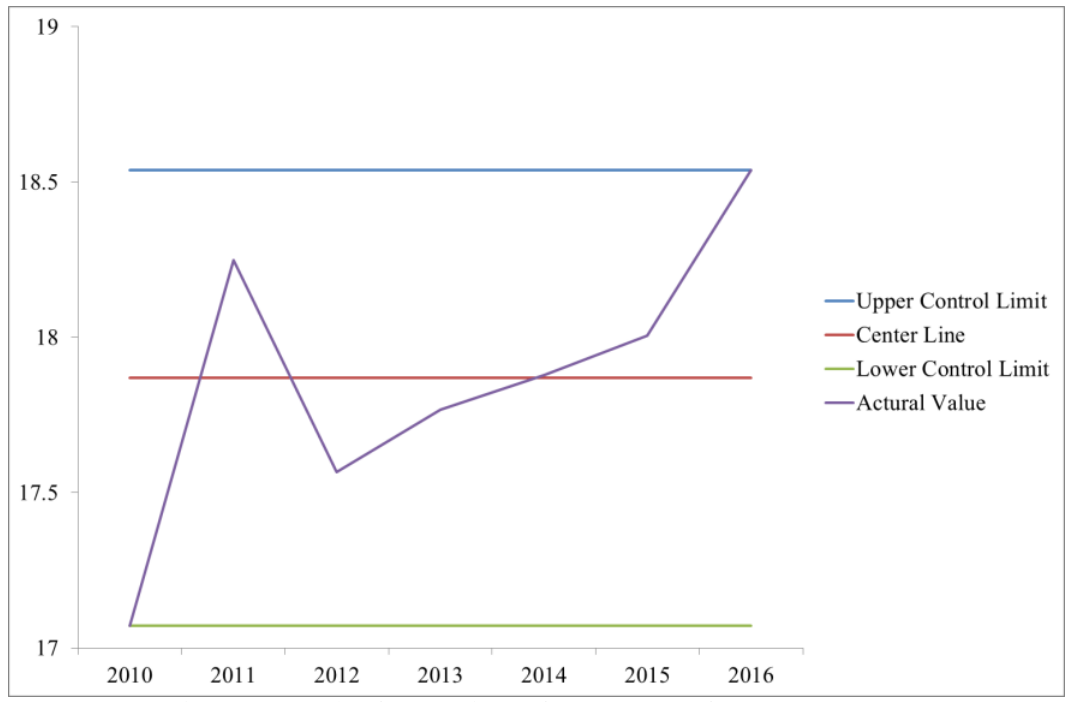

Figure 3. Trends of perceptions of management from 2010 to 2016 


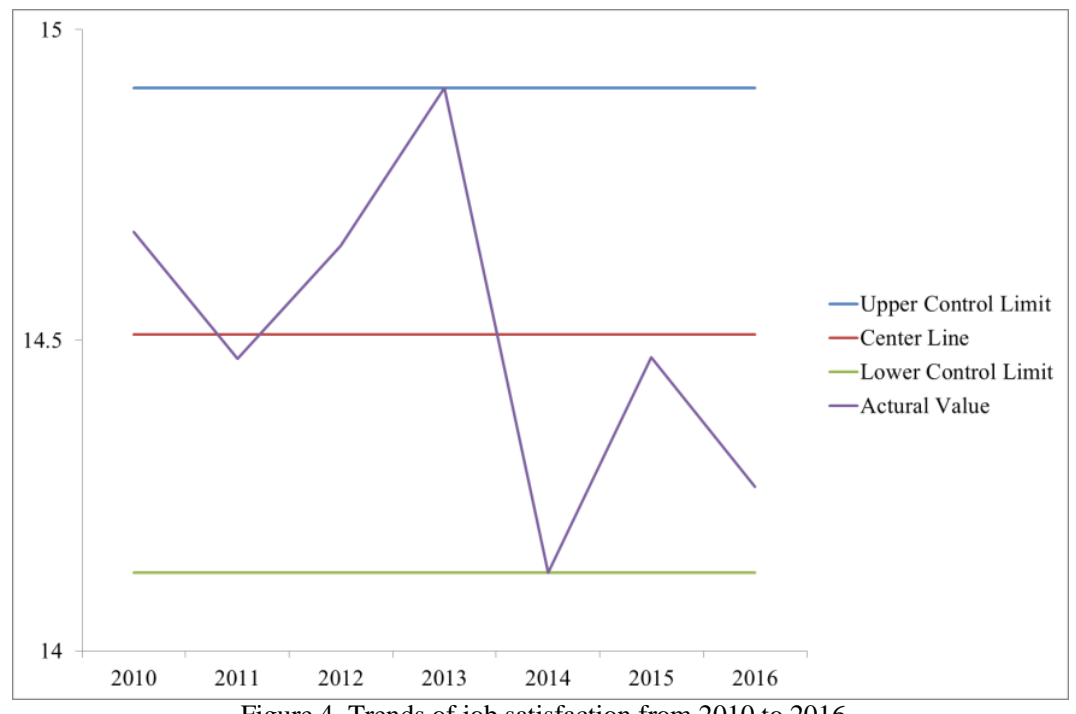

Figure 4. Trends of job satisfaction from 2010 to 2016

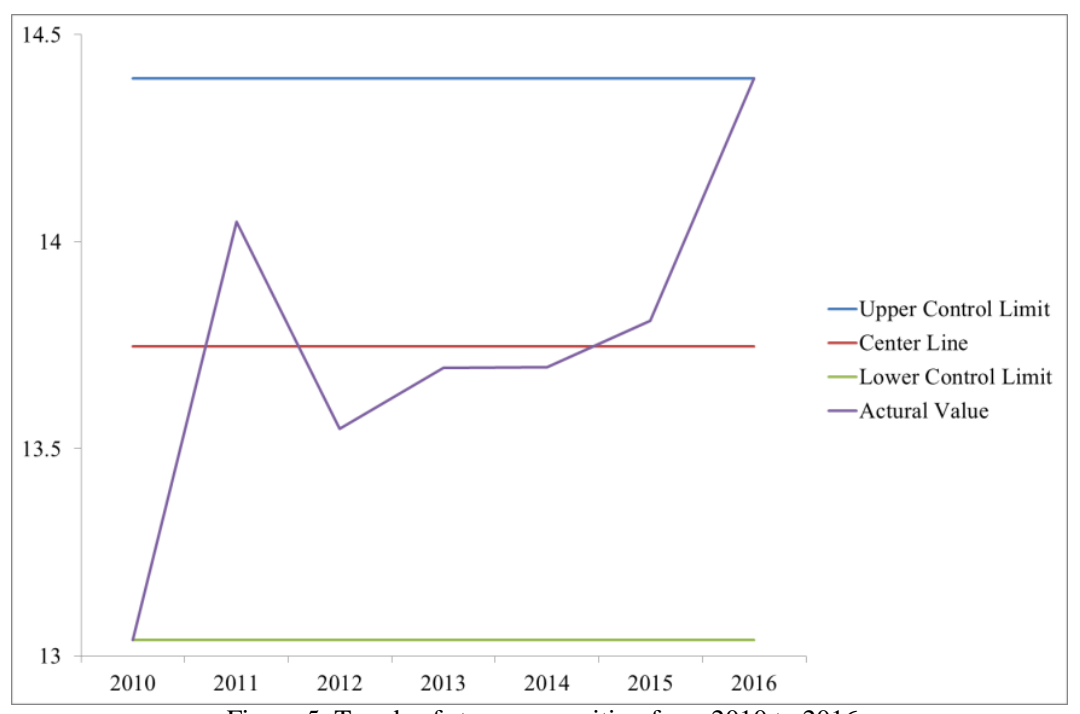

Figure 5. Trends of stress recognition from 2010 to 2016

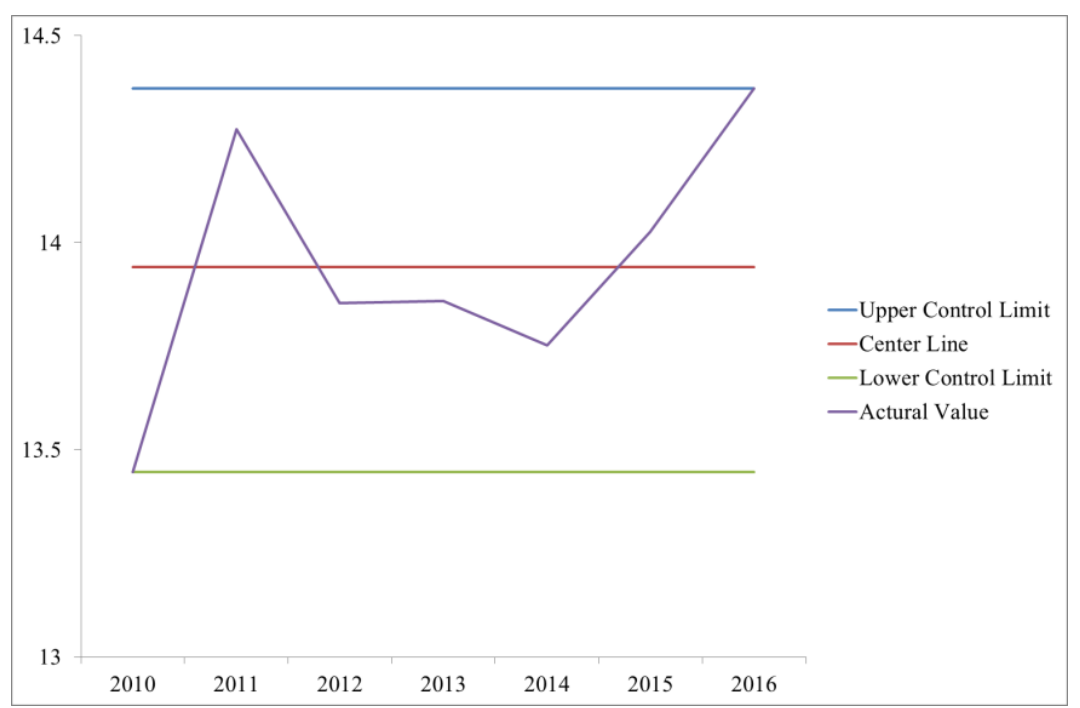

Figure 6. Trends of working conditions from 2010 to 2016 


\section{Conclusions}

Assessing the safety culture in healthcare organizations is essential. Monitoring the staff's attitudes toward safety culture regularly enables hospital management to understand current conditions, particularly the unsatisfied or non-ideal patient safety culture dimensions. The SAQ developed by Sexton et al. [5] is used to evaluate the safety culture of healthcare organizations in Taiwan on a yearly basis. Without a sufficient large sample size, the performance of each dimension cannot be determined to be improved, status quo, or deteriorated based on the performance each year. This study, on the other hand, proposes a framework using the bootstrap method to monitor the performance on a timely basis.

The focal point is not on the bootstrap method itself. In contrast, this study focuses on the philosophy to use the bootstrap method to generate control limits to determine the performance of each dimension each year based on a small sample size. Thus, hospital management can better understand how each dimension performs when the new results are available on a yearly basis. Moreover, when more new data are available, the control limits generated by the bootstrap method can be adjusted. The trends and changes for each dimension can be plotted in a control chart to allow hospital management to observe how the dimensions change over time. Finally, the philosophy can also be applied to other key performance indices in any healthcare organizations when the sample size is small to facilitate hospital management, compare the current scenario to the control limits generated by the bootstrap method, and quickly determine if the improvement action should be taken immediately.

\section{References}

1. J. Sorra, K. Khanna, N. Dyer, R. Mardon, and T. Famolaro, "Exploring Relationships Between Patient Safety Culture and Patients' Assessments of Hospital Care,” The Journal of Nursing Administration, Vol. 44, No. 10, pp. 45-53, September 2014

2. Y. C. Lee, H. H. Wu, W. L. Hsieh, S. J. Weng, L. P. Hsieh, and C. H. Huang, "Applying Importance-Performance Analysis to Patient Safety Culture," International Journal of Health Care Quality Assurance, Vol. 28, No. 8, pp. 1-15, 2015

3. O. Mazurenko, J. Richter, A. Swanson-Kazley, and E. Ford, "Examination of the Relationship Between Management and Clinician Agreement on Communication Openness, Teamwork, and Patient Satisfaction in the US Hospitals,"Journal of Hospital Administration, Vol. 5, No. 4, pp. 20-27, April 2016

4. G. N. Stock and K. L. McFadden, "Improving Service Operations: Linking Safety Culture to Hospital Performance," Journal of Service Management, Vol. 28, No. 1, pp. 57-84, 2017

5. J. B. Sexton, R. L. Helmreich, T. B. Neilands, K. Rowan, K. Vella, J. Boyden, et al., "The Safety Attitudes Questionnaire: Psychometric Properties, Benchmarking Data, and Emerging Research," BMC Health Services Research, Vol. 6, pp. 44, April 2006

6. C. Göras, F. Y. Wallentin, U. Nilsson, and A. Ehrenberg, "Swedish Translation and Psychometric Testing of the Safety Attitudes Questionnaire," BMC Health Services Research, Vol. 13, pp. 104, March 2013

7. G. Nguyen, N. Gambashidze, S. A. Ilyas, and P. Pascu, "Validation of the Safety Attitudes Questionnaire (Short Form 2006) in Italian in Hospitals in the Northeast of Italy," BMC Health Services Research, Vol. 15, pp. 284, July 2015

8. Y. C. Lee, S. C. Huang, C. H. Huang, and H. H. Wu, "A New Approach to Identify High Burnout Medical Staffs by Kernel kMeans Cluster Analysis in a Regional Teaching Hospital in Taiwan," Inquiry, Vol. 53, November 2016

9. Y. C. Lee, S. J. Weng, C. H. Huang, W. L. Hsieh, L. P. Hsieh, and H. H. Wu, "A Longitudinal Study of Identifying Critical Factors of Patient Safety Culture in Taiwan," Journal of Testing and Evaluation, Vol. 45, No. 3, pp. 1029-1044, May 2017

10. Y. C. Lee, J. I Shieh, C. H. Huang, C. Y. Wang, and H. H. Wu, "Analyzing Patient Safety Culture from Viewpoints of Physicians and Nurses-A Case of a Regional Teaching Hospital in Taiwan," Journal for Healthcare Quality, Vol. 39, No. 5, pp. 294-306, September 2017

11. Y. C. Lee, H. H. Wu, S. J. Weng, Y. C. Huang, W. L. Hsieh, and C. H. Huang, "Application of Hospital Information SystemsConstruction of an Incident Reporting System," The TEM Journal, Vol. 5, No. 4, pp. 530-537, November 2016

12. B. M. Smith and P. J. Gemperline, "Bootstrap Methods for Assessing the Performance of Near-Infrared Pattern Classification Techniques," Journal of Chemometrics, Vol. 16, No. 5, pp. 241-246, May 2002

13. A. Colubri, T. Silver, T. Fradet, K. Retzepi, B. Fry, and P. Sabeti, "Transforming Clinical Data into Actionable Prognosis Models: Machine-Learning Framework and Field-Deployable App to Predict Outcome of Ebola Patients," PLoS Neglected Tropical Diseases, Vol. 10, No. 3, pp. e0004549, March 2016

14. B. Efron and R. Tibshirani, "An Introduction to the Bootstrap," Chapman \& Hall, New York, 1994

15. C. H. Huang, H. H. Wu, and Y. C. Lee, "The Perceptions of Patient Safety Culture: A Difference Between Physicians and Nurses in Taiwan," Applied Nursing Research, Vol. 40, pp. 39-44, April 2018

16. European Union Network for Quality in Health Care, "Use of Patient Safety Culture Instrument and Recommendations," European Network for Quality in Health Care, Office for Quality Indicators, 2006

17. H. Johari, F. Shamsuddin, N. Idris, and A. Hussin, "Medication Errors Among Nurses in Government Hospital," Journal of Nursing and Health Science, Vol. 1, No. 2, pp. 18-23, February 2013

18. T. Leufer and J. Cleary-Holdforth, "Let's Do No Harm: Medication Errors in Nursing: Part 1," Nurse Education in Practice, Vol. 13, No. 3, pp. 213-216, May 2013 
19. Y. C. Lee, S. J. Weng, J. O. Stanworth, L. P. Hsieh, and H. H. Wu, "Identifying Critical Dimensions and Causal Relationships of Patient Safety Culture in Taiwan," Journal of Medical Imaging and Health Informatics, Vol. 5, No. 5, pp. 995-1000, September 2015

20. B. Efron, "Bootstrap Methods: Another Look at the Jackknife," The Annals of Statistics, Vol. 7, No. 1, pp. 1-26, January 1979

21. D. P. Kroese and J. C. C. Chan, "Statistical Modeling and Computation," Springer, New York, 2014

22. B. Goller, H. J. Pradlwarter, and G. I. Schuëller, "Robust Model Updating with Insufficient Data," Computer Methods in Applied Mechanics and Engineering, Vol. 198, No. 37-40, pp. 3096-3104, August 2009

23. D. Q. Li, X. S. Tang, and K. K. Phoon, "Bootstrap Method for Characterizing the Effect of Uncertainty in Shear Strength Parameters on Slope Reliability," Reliability Engineering \& System Safety, Vol. 140, pp. 99-106, August 2015

24. X. S. Tang, D. Q. Li, C. Zhou, and K. K. Phoon, "Bootstrap Method for Joint Probability Distribution Identification of Correlated Geotechnical Parameters," Rock and Soil Mechanics, Vol. 36, No. 4, pp. 913-922, April 2015

25. A. Yazdani, A. Nicknam, M. Khanzadi, and S. Motaghed, "An Artificial Statistical Method to Estimate Seismicity Parameter from Incomplete Earthquake Catalogs: A Case Study in Metropolitan Tehran, Iran,” Scientia Iranica A, Vol. 22, No. 2, pp. 400409, April 2015

26. M. R. Saberi, A. R. Rahai, M. Sanayei, and R. M. Vogel, "Steel Bridge Service Life Prediction using Bootstrap Method," International Journal of Civil Engineering, Vol. 15, No. 1, pp. 51-61, June 2017

27. S. J. Hsueh, Y. H. Hu, and C. H. Tu, "Economic Growth and Financial Development in Asian Countries: A Bootstrap Panel Granger Causality Analysis," Economic Modelling, Vol. 32, pp. 294-301, May 2013

28. J. Dlamini, M. Balcilar, R. Gupta, and R. Inglesi-Lotz, "Revisiting the Causality Between Electricity Consumption and Economic Growth in South Africa: A Bootstrap Rolling-Window Approach," International Journal of Economic Policy in Emerging Economies, Vol. 8, No. 2, pp. 169-190, 2015

29. K. H. You, S. A. Lee, J. K. Lee, and H. Kang, "Why Read Online News? The Structural Relationships Among Motivations, Behaviors, and Consumption in South Korea. Information," Communication \& Society, Vol. 16, No. 10, pp. 1574-1595, December 2013

30. D. Fu, Y. Hong, K. Wang, and W. Fan, "Effects of Membership Tier on User Content Generation Behaviors: Evidence from Online Reviews," Electronic Commerce Research, Vol. 18, No. 3, pp. 457-483, September 2018

31. M. R. Chernick and R. A. LaBudde, "An Introduction to Bootstrap Methods with Applications to R," John Wiley \& Sons, Hoboken, New Jersey, 2011 